\title{
Core-Satellite Nanomedicines for in Vivo Real-Time Monitoring of Enzyme-Activatable Drug Release by Fluorescence and Photoacoustic Dual-Modal Imaging
}

Xianlei Li, ${ }^{\dagger, \dagger, \otimes}$ Massimo Bottini, ${ }^{\dagger, \|, \otimes}$ Luyao Zhang ${ }^{\ddagger}, \#$ Shuai Zhang ${ }^{\nabla}$ Jing Chen ${ }^{\dagger}$ Tingbin Zhang ${ }^{\dagger}$

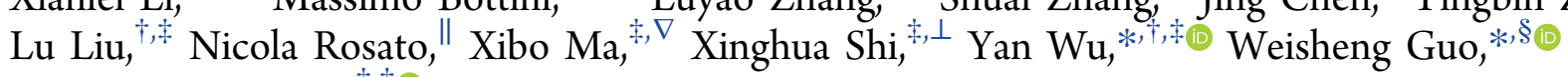
and Xing-Jie Liang*, ${ }^{*}$, \$0

${ }^{\dagger}$ CAS Key Laboratory for Biomedical Effects of Nanomaterials and Nanosafety, CAS Center for Excellence in Nanoscience, National Center for Nanoscience and Technology of China, No. 11, First North Road, Zhongguancun, Beijing 100190, P. R. China

${ }^{\ddagger}$ University of Chinese Academy of Sciences, Beijing 100049, P. R. China

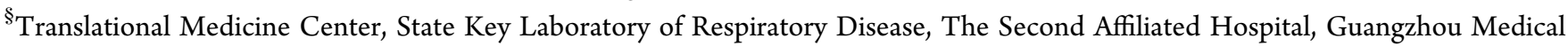
University, Guangzhou 510260, P. R. China

${ }^{\|}$Department of Experimental Medicine and Surgery, University of Rome Tor Vergata, Via Montpellier 1, 00133 Rome, Italy

${ }^{\perp}$ CAS Key Laboratory for Nanosystem and Hierarchy Fabrication, CAS Center for Excellence in Nanoscience, National Center for Nanoscience and Technology, Chinese Academy of Sciences, Beijing 100190, P. R. China

${ }^{\#}$ LNM, Institute of Mechanics, Chinese Academy of Sciences, Beijing 100190, P. R. China

${ }^{\nabla}$ CAS Key Laboratory of Molecular Imaging, Institute of Automation, Chinese Academy of Sciences, Beijing 100190, P. R. China

Supporting Information

ABSTRACT: It remains an unresolved challenge to achieve spatial and temporal monitoring of drug release from nanomedicines (NMs) in vivo, which is of crucial importance in disease treatment. To tackle this issue, we constructed coresatellite ICG/DOX@Gel-CuS NMs, which consist of gelatin (Gel) nanoparticles (NPs) with payloads of near-infrared fluorochrome indocyanine green (ICG) and chemo-drug doxorubicin (DOX) and surrounding CuS NPs. The fluorescence of ICG was initially shielded by satellite CuS NPs within the

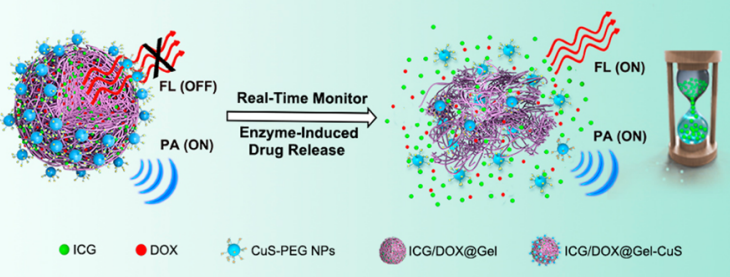
intact ICG/DOX@Gel-CuS NMs and increased in proportion to the amount of DOX released from NMs in response to enzyme-activated NMs degradation. For more comprehensive understanding of the drug-release profile, a theoretical model derived from computer simulation was employed to reconstruct the enzyme-activatable drug release of the ICG/DOX@Gel-CuS NMs, which demonstrated the underlying kinetics functional relationship between the released DOX amount and recovered ICG fluorescence intensity. The kinetics of drug release in vivo was assessed by administrating ICG/DOX@Gel-CuS NMs both locally and systemically into MDAMB-231 tumor-bearing mice. Upon accumulation of ICG/DOX@Gel-CuS NMs in the tumor, overexpressed enzymes triggered the degradation of the gelatin scaffold as well as the release of DOX and ICG, which can be visually depicted with the ICG fluorescence signal increasing only in the tumor area by fluorescence imaging. Additionally, the photoacoustic signal from CuS NPs was independent from the physical status of ICG/DOX@Gel-CuS NMs and hence was utilized for real-time NMs tracking. Thus, by taking advantage of the core-satellite architecture and NMs degradability in tumor site, the DOX release profile of ICG/DOX@Gel-CuS NMs was monitored by fluorescence and photoacoustic dual-modal imaging in a real-time noninvasive manner.

KEYWORDS: core-satellite, drug release in vivo, nanomedicines, computer simulation, dual-modal imaging

$\mathrm{T}$ he increasing interest in employing nanotechnology for the treatment of diseases has nurtured the development of drug delivery systems based on engineered nano-
Received: July 6, 2018

Accepted: December 28, 2018

Published: December 28, 2018 
Scheme 1. Development of Enzyme-Activatable ICG/DOX@Gel-CuS NMs with a Core-Satellite Architecture Made of Gelatin NPs Loaded with NIR-Fluorescent ICG and Chemo-Drug DOX, and Coated with "Satellite" CuS NPs"

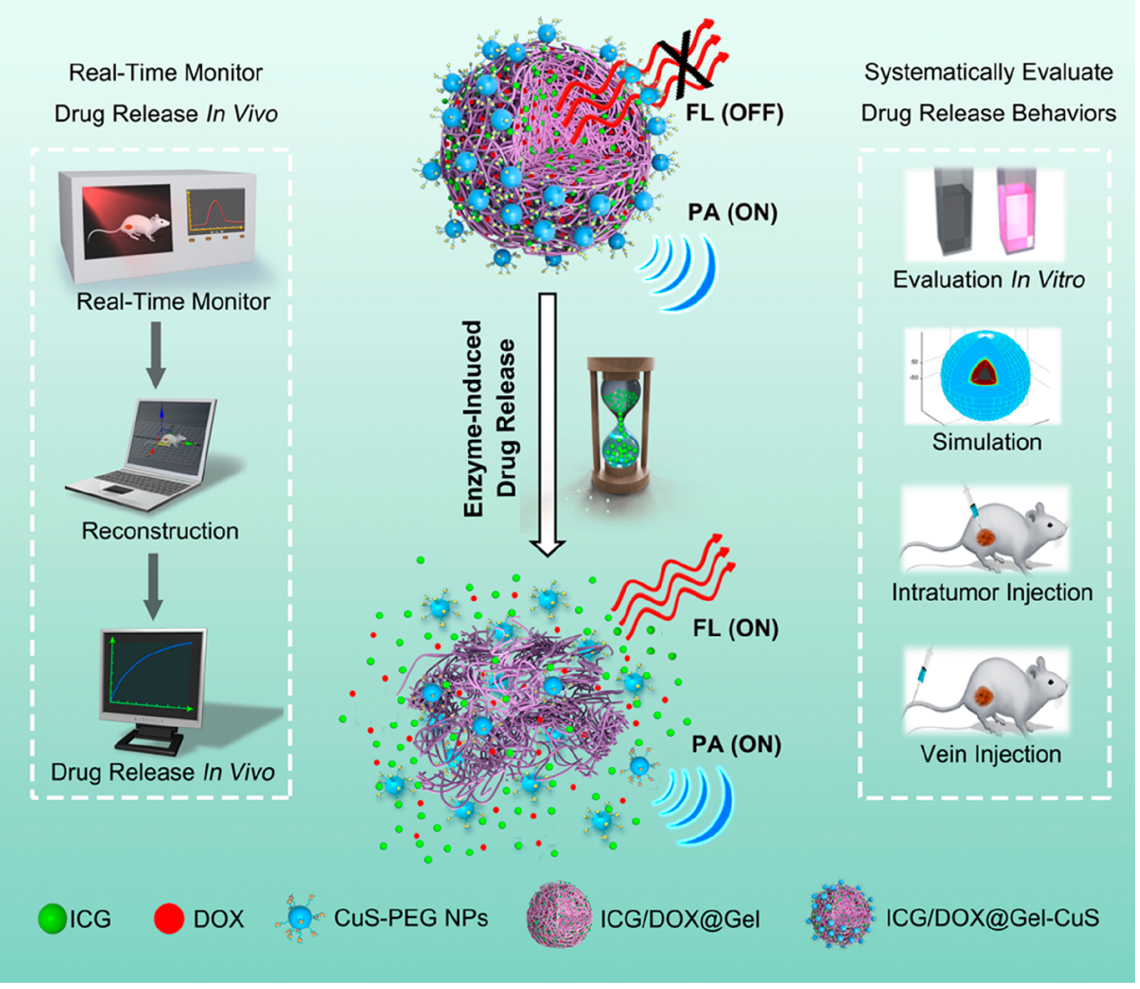

${ }^{a_{T}}$ The DOX release from ICG/DOX@Gel-CuS NMs can be monitored by fluorescence and photoacoustic (PA) dual-modal imaging in a real-time manner due to the core-satellite architecture and degradability in response to proteases overexpressed in tumor.

particles (NPs), also referred to as nanomedicines (NMs). ${ }^{1-6}$ Compared with traditional therapeutic approaches based on small molecules and biomacromolecules, NMs have proven distinctive advantages mainly at heightened accumulation and disease-site targeting delivery, benefiting from the discovery of enhanced permeability and retention (EPR) effects and their engineering surface. ${ }^{7-12}$ By incorporating the features of sitespecific delivery and reduced adverse side-effects, nanotherapies have showed great promise in clinical development, and several therapeutic NMs have been approved for clinical cancer treatments, including liposomes, polymeric micelles, and albumin NPs. ${ }^{13-17}$ Substantial preclinical research efforts on NMs have been mainly aimed at pursuing more efficient drug delivery by constructing multifunctional NMs to overcome the sequential biological barriers. ${ }^{18,19}$ However, it is noteworthy that the therapeutic efficacy of NMs is intensely associated with the drug release profiles as well. For instance, despite the higher accumulation of cisplatin loaded NMs (SPI-77) than free cisplatin in tumors, the phase III clinical trial of SPI-77 failed, which was largely attributed to the inadequate release and bioavailability of cisplatin. ${ }^{20}$ In addition, it is also highly desired in clinics to monitor the drug release quantitatively to realize precisely sufficient dosages, which is of great significance in decision making for individualized treatment with appropriate efficacy. ${ }^{21,22}$ Unfortunately, spatial-temporal drug release profiles of the NMs after administration has gained less attention and has been seldom investigated in depth. ${ }^{23-27}$

Most of the studies on the kinetics of drug release from NMs have been carried out by quantifying the drug released from NM dispersion in buffer solutions. Since the quantification of the drug release in vivo would be laborious and invasive, it is a common practice to label drugs with radionuclides or fluorochromes before entrapment into NMs and monitor their biodistibution by positron emission tomography (PET) or fluorescence (FL) imaging. ${ }^{28,29}$ Nevertheless, this approach cannot provide information about the kinetics of drug release from NMs. This problem has been typically tackled by directly labeling the loaded drug molecules and nanovehicles with different fluorophores, respectively. ${ }^{30,31}$ The drug release behaviors can be evaluated and reconstructed based on the quantitative and qualitative differences on the biodistributions between the loaded drugs and nanovehicles. Yet still the low signal/noise ratio and strong autofluorescence are strangling the precise drug release evaluations in vivo. Alternatively, subtle drug release would be instantaneously reflected by some responsive imaging signals, which is functionally associated with drug existence (released or entrapped). In this regard, a variety of stimuli-activatable NMs have been developed. ${ }^{32-34}$ However, the emerging stimuli-activatable NMs have been mostly based on external cues to trigger the release of the drug, which may hamper their translatability into clinics since the therapeutic testing and successive use on patients should be performed exclusively at clinics with special equipment. Therefore, it would be highly desirable to achieve real-time monitoring the drug released from NMs in response to internal stimuli displayed only by the diseased tissue.

Herein, we developed enzyme-activatable core-satellite ICG/DOX@Gel-CuS NMs, which consist of gelatin nanoparticles (NPs) loaded with the near-infrared (NIR) fluorescent indocyanine green (ICG) and chemo-drug doxorubicin (DOX), and decorated with "satellite" CuS NPs (Scheme 1). The in vitro study documented that the fluorescence of ICG was initially 


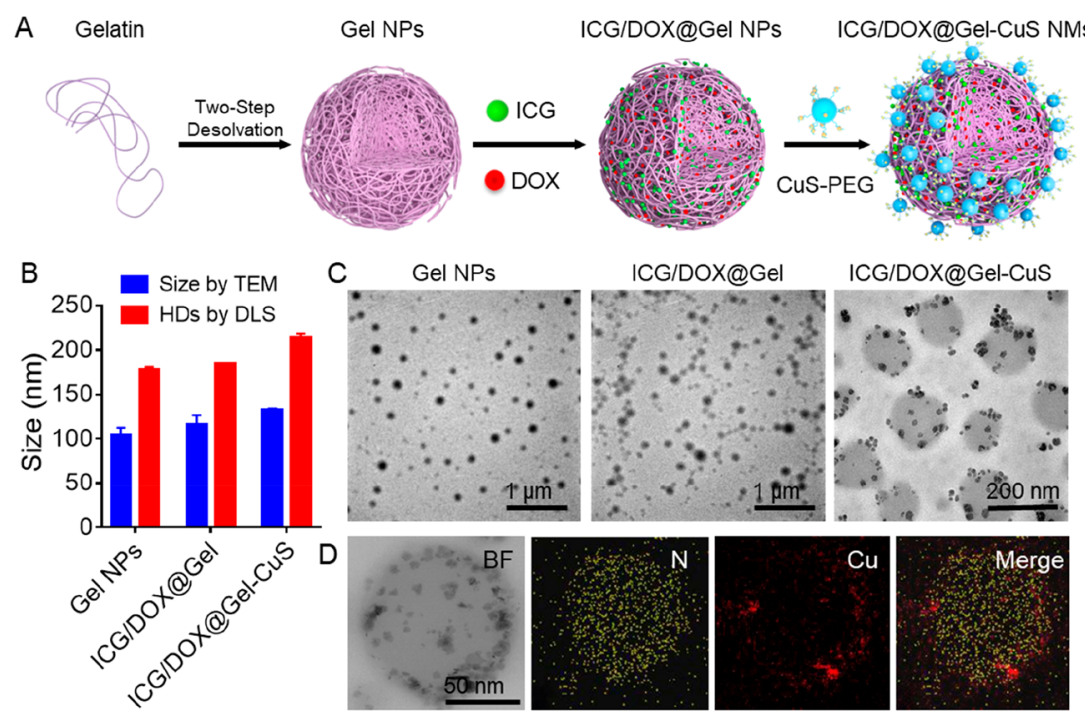

Figure 1. Fabrication and characterization of core-satellite ICG/DOX@Gel-CuS NMs. (A) Schematic illustration on the fabrication of ICG/ DOX@Gel-CuS NMs. (B) Diameter values (mean \pm standard error) of Gel NPs, ICG/DOX@Gel NPs and ICG/DOX@Gel-CuS NMs in hydrated state measured by TEM. (C) Representative TEM images of Gel NPs, ICG/DOX@Gel NPs, and ICG/DOX@Gel-CuS NMs. (D) Scanning transmission electron microscopy (STEM) imaging and corresponding elemental mapping (N and Cu) of the core-satellite ICG/ DOX@Gel-CuS NMs.

quenched within intact ICG/DOX@Gel-CuS NMs and increased in proportion to the amount of DOX released from ICG/DOX@Gel-CuS NMs due to the erosion of the gelatin matrix by protease. For more comprehensive understandings on the drug release profile, a theoretical model derived from computer simulation was employed to reconstruct the enzymeactivatable drug release of the ICG/DOX@Gel-CuS NMs, which scientifically demonstrated the underlying kinetics functional relationship between the released DOX amount and recovered ICG fluorescence intensity. The kinetics of drug release in vivo was assessed in tumor-bearing mice administrated with ICG/DOX@Gel-CuS NMs by in vivo fluorescence imaging system. Additionally, the photoacoustic signal arising from $\mathrm{CuS}$ NPs was not affected by the physical status of the ICG/DOX@ Gel-CuS NMs and thereby was used to monitor the biodistribution of the NMs. Thus, by taking advantage of the core-satellite architecture and NMs degradability in tumor site, the DOX release profile of ICG/DOX@Gel-CuS NMs was monitored by fluorescence and photoacoustic dual-modal imaging in a real-time noninvasive manner.

\section{RESULTS AND DISCUSSION}

Fabrication and Characterization of ICG/DOX@GelCuS NMs. Core-satellite ICG/DOX@Gel-CuS NMs were made of gelatin NPs (Gel NPs) loaded with ICG and DOX, and coated with polyethylene glycol (PEG)-modified CuS NPs. As depicted in Figure 1A, Gel NPs were first fabricated via a modified two-step desolvation method. ${ }^{35}$ ICG and DOX were then loaded into the Gel NPs under sonication to obtain the ICG/DOX@Gel NPs. Finally, core-satellite ICG/DOX@Gel$\mathrm{CuS}$ NMs were obtained by grafting $10 \mathrm{~nm}$ in diameter PEGmodified CuS NPs (Figure S2) onto the surface of ICG/DOX@ Gel NPs via a carboxyl-amine coupling strategy. Noticeably, for the coupling chemistry of PEGylated CuS NPs to the ICG/ DOX@Gel NPs, the sequence that carboxy-activated ICG/ DOX@Gel NPs should be added dropwise into CuS solution would not be reversable to avoid cross-linking. Transmission electron microscopy (TEM) imaging and dynamic light scattering (DLS) analysis showed that Gel NPs had a spherical shape with a diameter in the dehydrated state (DD) of $104.1 \pm$ $8.7 \mathrm{~nm}$ and a hydrodynamic diameter (HD) of $176.2 \pm 2.7 \mathrm{~nm}$ with a polydispersity index (PDI) of $0.113 \pm 0.009$ (Figures 1B,C). The DD and HD of the obtained ICG/DOX@Gel NPs were $116.4 \pm 10.3 \mathrm{~nm}$ and $184.0 \pm 0.9 \mathrm{~nm}$, respectively (Figure 1B,C). ICG/DOX@Gel-CuS NMs showed a core-satellite morphology with a DD of $132.0 \pm 1.24 \mathrm{~nm}$ as measured by TEM (Figure 1C) and a HD of $213.9 \pm 4.5 \mathrm{~nm}$ measured by DLS (Figure 1B). In addition, mouse serum containing PBS was employed to simulate blood, and we tested the cumulative release of ICG and DOX from the ICG/DOX@Gel-CuS NMs by incubating the NMs into the serum $(10 \%)$ containing PBS. As shown in Figure S3, neither DOX nor ICG showed obvious release from the NMs up to 6 days, suggesting mere leakage of the cargo and the fairly good structural stability of the NMs under physiological circumstances. Scanning transmission electron microscopy (STEM) imaging and elemental mapping analysis (Figure 1D) were performed to further validate the spherical and core-satellite morphology of the ICG/DOX@ Gel-CuS NMs.

Evaluation of DOX Release from ICG/DOX@Gel-CuS NMs in Vitro. We tested the changes in the morphological and spectroscopic properties of core-satellite ICG/DOX@Gel-CuS NMs upon trypsin addition to assess the capacity of ICG/ DOX@Gel-CuS NMs for real-time monitoring and quantification of enzyme-activated drug release. As illustrated in Figure $2 \mathrm{~A}$, the addition of trypsin led to efficient structural degradation of the ICG/DOX@Gel-CuS NMs, which was verified by TEM observation in Figure 2B and Figure S4. In the absence of trypsin, the emission spectrum of the core-satellite ICG/ DOX@Gel-CuS NMs dispersion showed an unchanged faint peak at $815 \mathrm{~nm}$, suggesting that the satellite CuS NPs almost completely quenched the fluorescence arising from the ICG molecules entrapped within the gelatin matrix (Figure 2C and Figure S5). Upon addition of trypsin, the fluorescence intensity of the dispersion of ICG/DOX@Gel-CuS NMs increased gradually (Figure 2D,E). These lines of evidence suggested that 

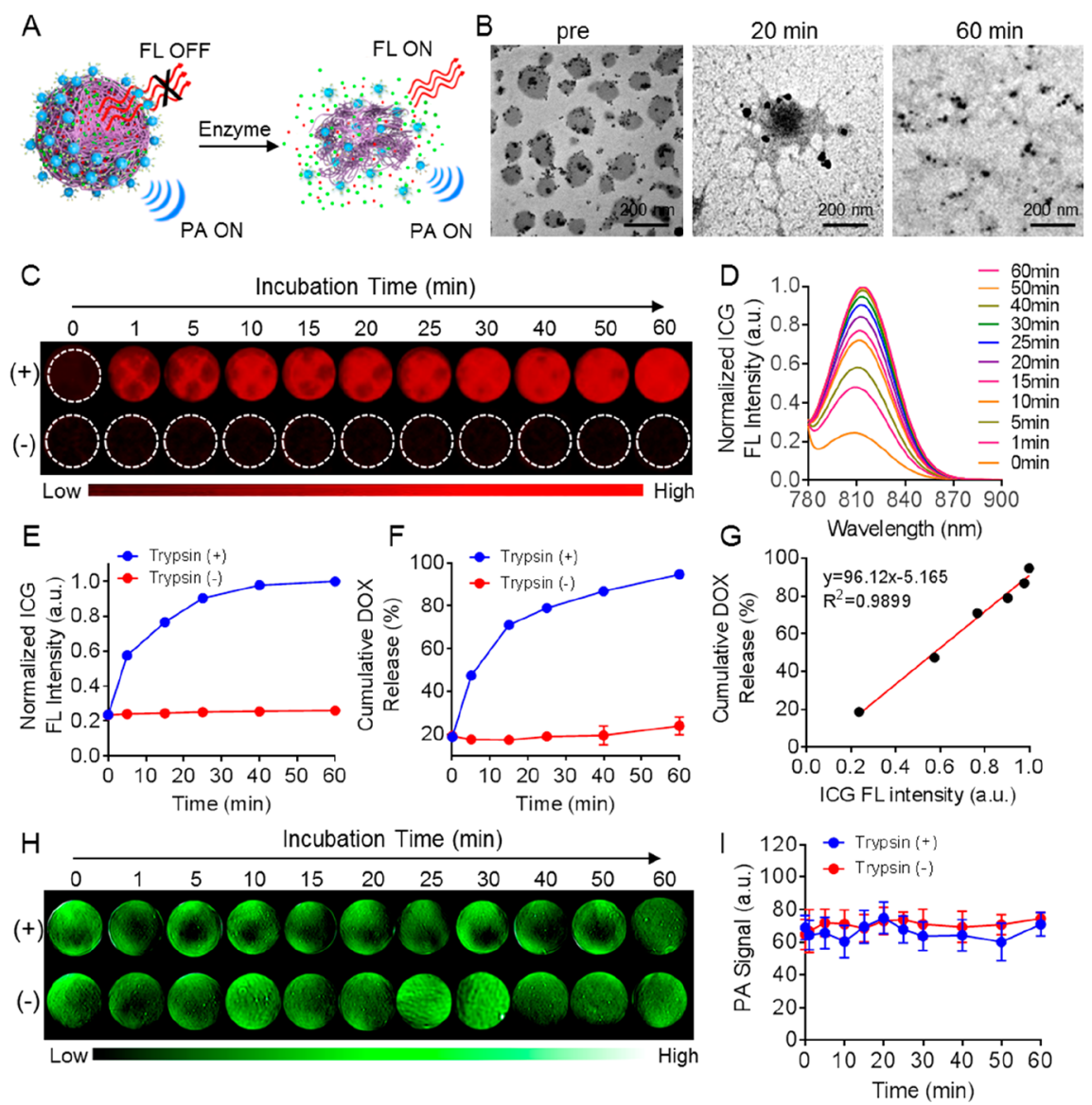

Figure 2. Evaluation of drug release from ICG/DOX@Gel-CuS NMs dispersion in PBS. (A) Core-satellite architecture of ICG/DOX@GelCuS NMs enables real-time monitoring and quantification of enzyme-activated DOX release by fluorescence/PA dual-modal imaging. (B) TEM images of core-satellite ICG/DOX@Gel-CuS NMs before (pre) and after the addition of trypsin. (C) Fluorescence images recorded for coresatellite ICG/DOX@Gel-CuS NMs dispersions in the absence $(-)$ and presence $(+)$ of trypsin at different times. (D) Emission spectra of ICG/ DOX@Gel-CuS NMs dispersion in PBS after the addition of trypsin. (E) Intensity of the $815 \mathrm{~nm}$ emission peak of the ICG/DOX@Gel-CuS NMs dispersion in the absence $(-)$ and presence $(+)$ of trypsin at different times. (F) Time-dependent profile of cumulative release of DOX from ICG/DOX@Gel-CuS NMs in the absence (-) and presence $(+)$ of trypsin measured by UV-vis spectrophotometer. (G) Linear fit between the percentage of released DOX and the fluorescence intensity of ICG in the presence of trypsin. (H) PA images and (I) quantitative analysis of ICG/DOX@Gel-CuS NMs dispersions in the absence (-) and presence $(+)$ of trypsin at different times.

the observed enzyme-triggered recovery of ICG/DOX@Gel$\mathrm{CuS}$ NMs fluorescence was due to the concomitant shedding of the CuS NPs off the NMs' surface and release of ICG from the NMs. The DOX released from the ICG/DOX@Gel-CuS NMs was collected after incubation with trypsin at different times and quantified by UV-vis spectrometry (Figure 2F). The cumulative release of DOX followed biexponential kinetics with approximately $71 \%$ of the DOX release during the first 15 min of incubation with trypsin and an additional $24 \%$ release in the following period were found. However, it remains a question whether the fluorescence signal change could be used as a quantitative reporter of DOX release. A correlation study was thereby performed, which indicated that the fluorescence intensity of ICG $(x)$ had a nearly linear relationship with the cumulative amount of released DOX $(y)$ (Figure 2G). The correlation was well fitted as a linear equation: $y=96.12 x-$ $5.165\left(R^{2}=0.9899\right)$. This result documented that the DOX released from ICG/DOX@Gel-CuS NMs could be readily monitored by detecting the fluorescence intensity of the ICG in a real-time quantitative manner. The photoacoustic (PA) signal of the ICG/DOX@Gel-CuS NMs dispersion in PBS was also investigated in the absence/presence of trypsin at different times. As presented in Figure 2H,I, the PA signal was not affected by the presence of the enzyme, which enables the realtime monitoring of NMs biodistribution by PA imaging. Taken together, these results validated that the core-satellite ICG/ DOX@Gel-CuS NMs could realize real-time monitoring and quantification of enzyme-activated DOX release by a combination of fluorescence and PA imaging.

Mathematical Model of Drug Release from EnzymeActivatable NMs via Computer Simulation. For insightful scientific exploration the kinetics of DOX and ICG release from the enzyme-activatable ICG/DOX@Gel-CuS NMs, a mathematical model was established by computational simulation, where both the processes of NMs erosion and drug diffusion were taken into consideration. We hypothesized that the erosion of the NMs is carried out by enzymes permeating the degradable matrix and facilitates the successive diffusion of the molecules (ICG and DOX) from the matrix of the NMs into the surrounding space. The diffusion process can be described by the equation

$$
\frac{\partial c}{\partial t}=\nabla(D \nabla c)
$$

where $c$ is the drug concentration and $D$ is the diffusion constant in the matrix of the ICG/DOX@Gel-CuS NMs. D can be 

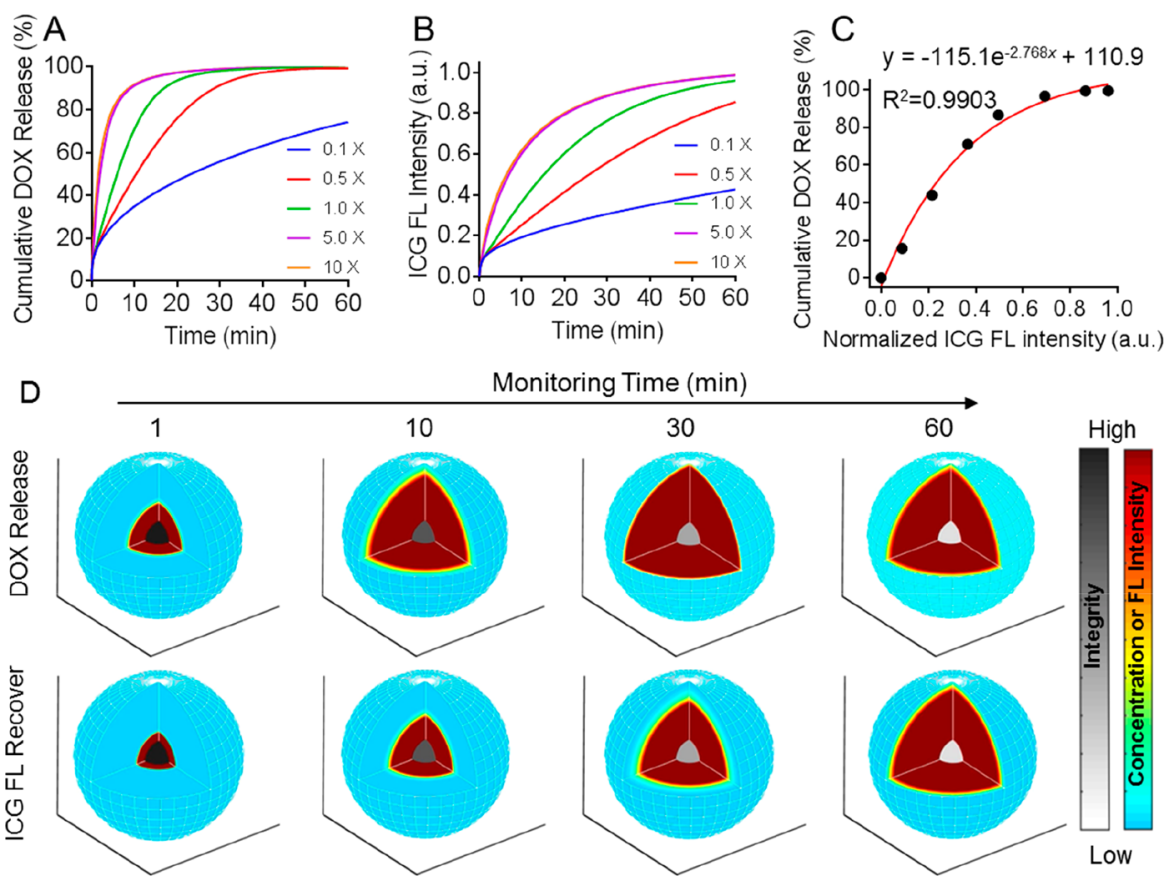

Figure 3. Mathematical model of DOX release and ICG fluorescence recover from enzyme-activatable NMs via computer simulation. (A) DOX release derived for different values of $\lambda$ (see eq 11). (B) Fluorescence recovery of ICG by computational simulation. (C) One phase decay fitting between the percentage of released drug (DOX) and the recovered fluorescence intensity (ICG). (D) Frames from movie S1 and movie S2 at different times. The inner and outer spheres exemplify a gelatin-based NM and the surrounding environment, respectively. The process of NMs degradation with time is shown by coloring the inner sphere with decreasing shades of gray, whereas the process of DOX diffusion and ICG fluorescence recover in the surrounding environment is shown by coloring the outer sphere with the colors of the visible spectrum.

expressed as a function of the diffusion constant $D_{0}$ in the surrounding environment and the volume percentage of the matrix of the ICG/DOX@Gel-CuS NMs according to the free volume theory $^{36-38}$

$$
D=D_{0}\left(1-k_{1} r_{\mathrm{s}} \varphi^{0.75}\right) \exp \left(-k_{2} r_{\mathrm{s}}^{2} \frac{\varphi}{1-\varphi}\right)
$$

where $k_{1}$ and $k_{2}$ are two constants related to the physical and chemical properties of the matrix of the NMs, $r_{s}$ is the typical molecular radius of the entrapped drug, and $\varphi$ is the volume percentage of the matrix of the NMs, which is related to the process of erosion. According to a first-order kinetic model, which is a simplified version of the classical Michaelis-Menten model, ${ }^{39} \varphi$ can be calculated by the equation

$$
\frac{d \varphi}{d t}=-k_{3} c_{\mathrm{e}} \varphi
$$

where $k_{3}$ is the diffusion constant and $c_{\mathrm{e}}$ is the enzyme concentration. By considering an initial volume percentage of the matrix equal to $90 \%$ of the total volume of the NMs, eq 3 can be solved as

$$
\varphi=0.9 \exp \left(-k_{3} c_{\mathrm{e}} t\right)
$$

The diffusion process can be divided into two parts, one describing the diffusion of the drug through the matrix

$$
\frac{1}{D} \frac{\partial c}{\partial t}=\frac{\partial^{2} c}{\partial t}+\frac{2}{r} \frac{\partial c}{\partial r}
$$

where $D$ satisfies the eq 2 and the other describing the diffusion of the drug in the surrounding environment:

$$
\frac{1}{D_{0}} \frac{\partial c}{\partial t}=\frac{\partial^{2} c}{\partial t}+\frac{2}{r} \frac{\partial c}{\partial r}
$$

The flux of the drug in the matrix and surrounding environment had the same value at the interface:

$$
\frac{1}{D_{0}} \frac{\partial c}{\partial t}=\frac{1}{D} \frac{\partial c}{\partial t}
$$

Additionally, at $t=0$

$$
\begin{aligned}
& c(r, 0)=c_{0} \text { at } r \leq R \\
& c(r, 0)=c_{0} \text { at } r>R
\end{aligned}
$$

where $R$ is the radius of the NMs. At the average distance among NMs $\left(r=R_{\mathrm{b}}\right)$, the flux of the drug was assumed to be zero:

$$
\frac{\partial c}{\partial r}=0 \text { at } r=R_{\mathrm{b}}
$$

In order to simplify the solution of the problem, the following parameters were chosen to make the equation dimensionless:

$$
\begin{aligned}
& \bar{t}=k_{3} c_{\mathrm{e}} t \\
& \bar{r}=r \sqrt{k_{2}} \\
& \lambda=\frac{k_{3} c_{\mathrm{e}}}{D_{0} k_{2}} \\
& \beta=k_{2} r_{\mathrm{s}}^{2} \\
& \gamma=k_{1} r_{\mathrm{s}}
\end{aligned}
$$




$$
\bar{D}_{0}=\frac{D_{0} k_{2}}{k_{3} c_{\mathrm{e}}}
$$

The system of eqs 5 and 6 is general and can be solved to derive the profile of drug release from enzyme-activatable NMs. We solved the system by MATLAB and derived the profile of ICG fluorescence recovery and DOX release from the gelatinbased NMs. As shown in Figure 3A, the NMs possessed distinct release behaviors for different values of $c_{\mathrm{e}}$. In order to compare the kinetics of DOX release from ICG/DOX@Gel-CuS NMs dispersed in PBS with the simulation data (Table S1), we calculated the values of the correlation parameters for $c_{\mathrm{e}}=2.5 \times$ $10^{-5} \mathrm{~g} / \mathrm{mL}\left(\lambda=1.8 \times 10^{-6}\right)$, which corresponded to the concentration of trypsin added to the dispersion of ICG/DOX@ Gel-CuS NMs. Noticeably, the derived kinetics of DOX release showed a sigmoidal behavior, and the simulation data showed that approximately $98 \%$ of drug is released from the NMs after $30 \mathrm{~min}$ in the presence of $2.5 \times 10^{-5} \mathrm{~g} / \mathrm{mL}$ of trypsin. The drug release rate of the NMs significantly decreased at lower enzymatic concentrations (red and blue curves in Figure 3A). With regard to the ICG fluorescence recovery model, we simulated its diffusion or fluorescence process with parameters at Table $\mathrm{S} 2$ which satisfies the same function as DOX. As shown in Figure 3B, the fluorescence recovery of ICG presented a similar profile to the release behavior of DOX. Furthermore, the correlation function between the percentage of released DOX and the recovered fluorescence of ICG was well fitted as one phase decay $\left(y=-115.1 \mathrm{e}^{-2.768 x}+110.9, R^{2}=0.9903\right)$ (Figure 3C). It also confirmed that the core-satellite ICG/DOX@Gel$\mathrm{CuS}$ NMs can act as real-time monitoring drug (DOX) release behaviors via the fluorescence turn-on process of ICG, which mutually supports the results in vitro in Figure 2.

The data derived from the computational simulation were manipulated through computer graphics and imaging processing techniques to reconstruct the three-dimensional (3D) drug release process of ICG/DOX@Gel-CuS NMs in movies (movie S1 and movie S2). Four representative frames of the movies depicting the DOX release and ICG fluorescence recovery are presented in Figure 3D. Both the ICG/DOX@Gel-CuS NMs structure erosion and drug diffusion into the surrounding environment were considered and displayed. In this model, we considered the molecule to diffuse radially starting from the surface of the NMs for simplicity. It is worth noting that the grid edge of the sphere in Figure 3D does not indicate the constant size of the NMs and refers to the diffusion space outside the NMs instead. The degradation of the NMs in response to enzyme was indexed by the integrity change from intact status (in black) gradually to dissociated status (in white). The concentration of the released DOX or the recovered fluorescence intensity of ICG within the diffusion space was indexed by the color change from cyan (zero) to red (high concentration or high fluorescence intensity).

Real-Time Monitoring of DOX Release from ICG/ DOX@Gel-CuS NMs in Vivo. On the basis of the favorable results obtained with NMs dispersions and computer simulation studies, we tested the core-satellite ICG/DOX@Gel-CuS NMs as a reporter for real-time monitoring and quantification of enzyme-activated drug release in vivo. Prior to evaluation on the drug release behaviors in vivo, the tumor-site accumulation profile of ICG/DOX@Gel-CuS NMs administered via tail vein injection was assessed by multispectral optoacoustic tomography (MSOT) because the PA signal from $\mathrm{CuS}$ NPs was independent from the physical status of ICG/DOX@Gel-CuS
NMs (Figure 2H,I). Upon systemic administration, the PA signals in the tumor sites were gradually enhanced and persisted for up to $24 \mathrm{~h}$ (Figure 4A,B), indicating the efficient

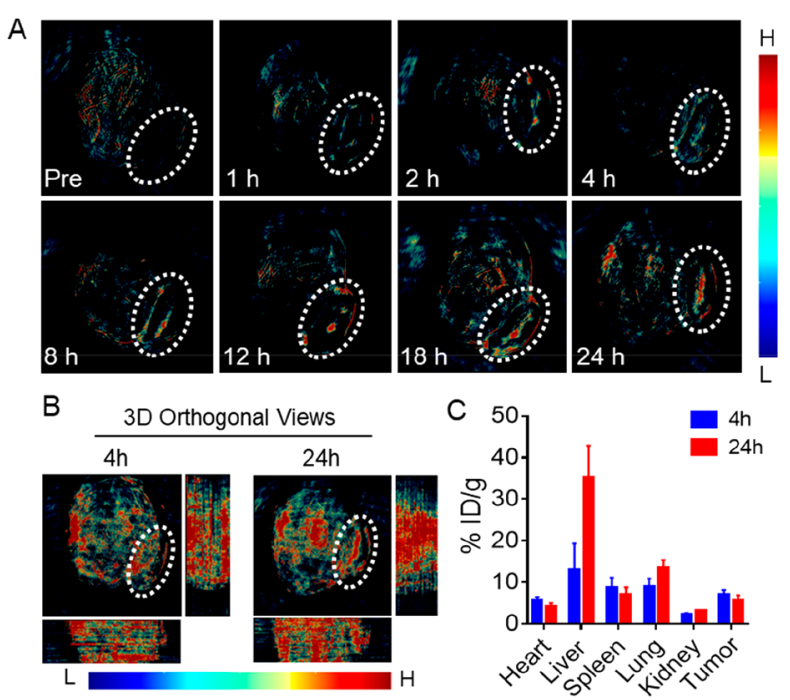

Figure 4. Tumor site accumulation monitoring of ICG/DOX@GelCuS NMs in vivo. (A) In vivo MSOT images of mice injected with ICG/DOX@Gel-CuS NMs at different time points postinjection (p.i.). (B) Orthogonal MSOT images of the tumor region at 4 and 24 h p.i. (C) Biodistribution of ICG/DOX@Gel-CuS NMs at 4 and $24 \mathrm{~h}$ after systematical injections measured by inductively coupled plasma mass spectrometry (ICP-MS).

accumulation of ICG/DOX@Gel-CuS NMs in tumors by exploiting the EPR effect. At 4 and 24 h postinjection (p.i.), the major organs of the treated mice were collected and the biodistribution quantification of the ICG/DOX@Gel-CuS NMs was determined on the basis of the percentage of injected dose per tissue gram (\% ID/g) of copper element by inductively coupled plasma mass spectrometry (ICP-MS). As depicted in Figure 4C, the ICG/DOX@Gel-CuS NMs showed fairly high tumor-site accumulation efficiency, which was $7.02 \% \mathrm{ID} / \mathrm{g}$ and $5.66 \% \mathrm{ID} / \mathrm{g}$ at 4 and $24 \mathrm{~h}$ p.i., respectively. The high tumor uptake efficiency of the ICG/DOX@Gel-CuS NMs is due to the EPR effect, and this implies that PA imaging can be exploited for real-time tracking the accumulation behavior of ICG/DOX@ Gel-CuS NMs in a tumor microenvironment.

Next, we sought to compare the kinetics of ICG fluorescence recovering of ICG/DOX@Gel-CuS NMs in the presence and absence of enzyme in vivo. It has been documented that the tumor microenvironment showed endogenous characteristic of high concentrations of matrix metalloproteinases (MMPs), which are efficient at hydrolyzing gelatin. ${ }^{40-42}$ Thus, the tumorbearing mouse received an intratumoral local injection of ICG/ DOX@Gel-CuS NMs and a subcutaneous injection with an equal dose at the opposite side. The NIR fluorescence signals arising from the areas around the injection points were recorded and quantified by the IVIS fluorescence imaging system. As shown in Figure 5A, significant distinct fluorescence signals were clearly observed in the tumor site after the injection, while only faint fluorescence was detected at the opposite site throughout the observation up to $4 \mathrm{~h}$. The quantification analysis in Figure $5 \mathrm{~B}$ revealed that the average fluorescence signal arising from the tumor area markedly increased during the starting $60 \mathrm{~min}$ p.i., a pattern similar to the in vitro fluorescence profile of the ICG/ DOX@Gel-CuS NMs dispersion in the presence of trypsin 

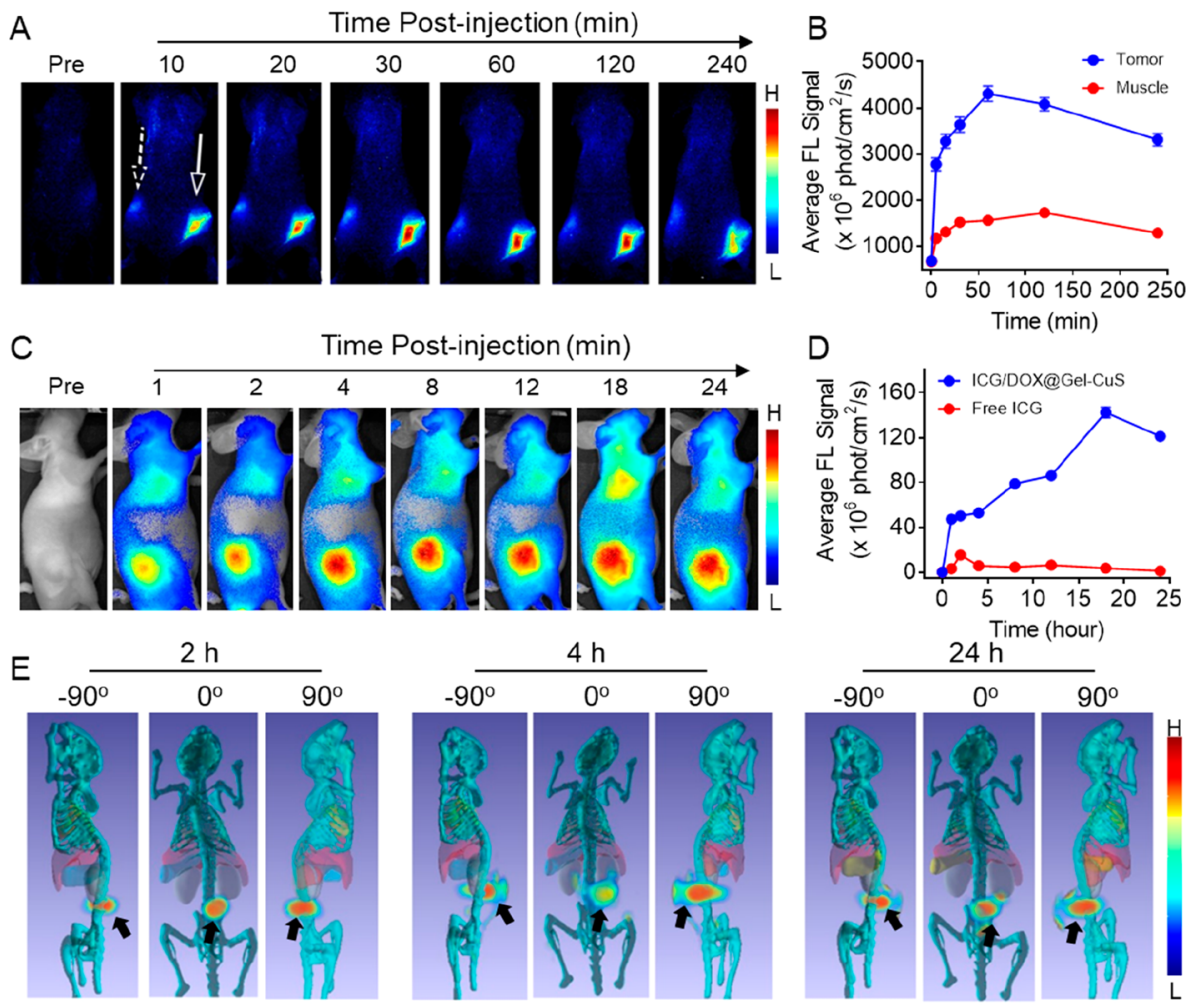

Figure 5. Evaluation of DOX release from ICG/DOX@Gel-CuS NMs in vivo. (A) In vivo NIR fluorescence images of mice treated with NMs via both subcutaneous (dotted arrow) and intratumor (solid arrow) injection at different times postinjection. (B) Quantitative data (mean \pm standard error) of the NIR signal emitted from the injection areas shown in (A). (C) NIR fluorescence images of mice treated with ICG/DOX@ Gel-CuS NMs via tail vein injection at different times postinjection. (D) Quantitative data (mean \pm standard error) of the NIR signal emitted from the injection area shown in (C). (E) 3D-reconstruction of transillumination fluorescence images of mice treated with NMs via tail vein injection at various tilt angles.

(Figure 2E). The following slight decrease of the fluorescence after $1 \mathrm{~h}$ should be attributed to the gradual diffusion and clearance of ICG from the tumor site. On the contrast, the NIR signal arising from the area around the subcutaneous injection showed a very faint increase in intensity. These results indicated that the core-satellite ICG/DOX@Gel-CuS NMs could efficiently degrade in the tumor region and persist intact in the healthy tissue. Given the linear function between the percentage of released DOX and the fluorescence intensity of ICG (Figure $2 \mathrm{G}$ ) and the kinetics in vivo similar to that of NMs in vitro (Figure 5B), it is reasonable to derive the kinetic of DOX release in vivo from the recorded fluorescence kinetic of ICG. The derived kinetic curve of DOX release in vivo was displayed in Figure S7A, which suggested that $56.8 \%$ of DOX was released in the tumor microenvironment during the first $5 \mathrm{~min}$ postinjection in vivo compared to $\sim 47.4 \%$ of DOX released in PBS. It might be indicative of a stronger burst-release in vivo than in solution most likely due to a higher concentration of proteases able to degrade the gelatin matrix.

Considering the clinical relevance of the systemic administration of anticancer drugs, we assessed the kinetic profile of drug release of ICG/DOX@Gel-CuS NMs, which were injected into tumor-bearing mice via tail vein. A strong increase in the NIR fluorescence intensity was observed in the tumor area but not in other tissues, which correlated very well with the overexpression of proteases in tumors in respect to normal tissues (Figure 5C). Quantitative analysis revealed that the NIR emission arising from the tumor area significantly increased until $18 \mathrm{~h}$ p.i. and then gradually decreased (Figure 5D), which is intensely dependent on the tumor-site accumulation and drug release kinetic of the ICG/DOX@Gel-CuS NMs. Similarly, the kinetic profile of DOX release in vivo was also calculated from the fluorescence signal of ICG following the established linear equation and plotted in Figure S7B. In order to gain more information about the spatial distribution of the fluorescence and drug release, 3D transillumination fluorescence imaging analysis was carried out at $2 \mathrm{~h}, 4$ and $24 \mathrm{~h}$ p.i. Compared to the 2D fluorescence imaging with plane projection, 3D-reconstruction transillumination fluorescence feedbacks the signal in depth of tumor, as well the penetration ability of ICG (Figure 5E, movies S3-S5). The NIR signal arose from deep regions of the tumor, combining the result of MSOT imaging (Figure 4A,B) which indicated the penetration ability of $\mathrm{CuS}$, validating the ability of the core-satellite NMs to egress from the angiogenic blood vessels by exploiting the EPR effect and release drugs to penetrate into the tumor parenchyma. To further evaluate the drug release behaviors, major organs and tumor of the mice were collected at $24 \mathrm{~h}$ p.i. for ex vivo fluorescence analysis (Figure S8 and S9). The NIR emission was much stronger in the tumor than other tissues, which was explained by a more efficient degradation of NMs in tumors due to a greater concentration of proteases. In line of the evidence, it illustrated that the enzymeactivatable drug release behavior of the core-satellite ICG/ DOX@Gel-CuS NMs can be monitored by fluorescence/PA dual-modal imaging in a real-time and semiquantitative manner.

Antitumor Effect Evaluation Benefiting from Drug Release. Given the imaging guided enzyme-activatable drug release in vivo of ICG/DOX@Gel-CuS NMs and the systemic 

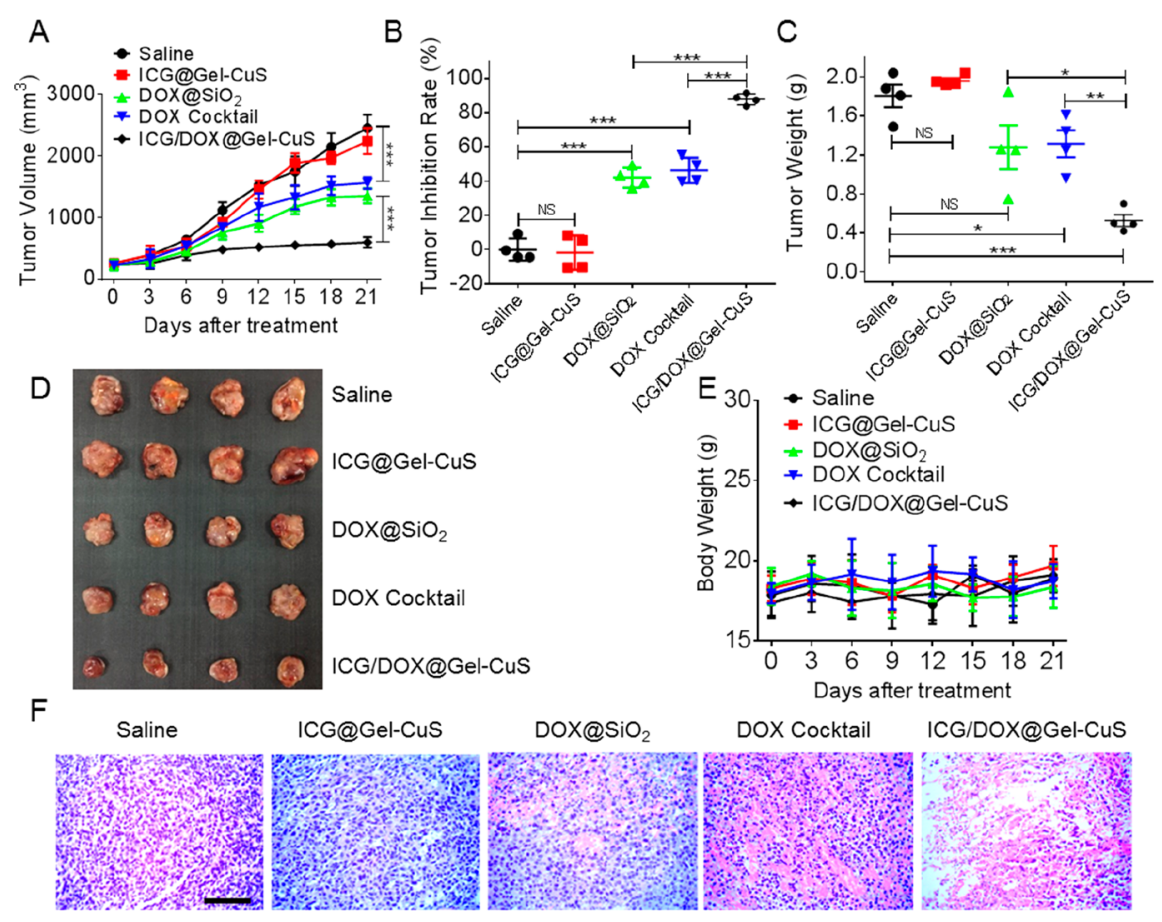

Figure 6. Anticancer therapeutic effect evaluation in vivo. (A) Tumor volumes of different groups were measured and calculated every 3 days. (B) Tumor inhibition rate of different groups. (C) Tumor weight measured at the end of treatment. (D) Pictures of tumors at the end of recording time. (E) Body weight curves of mice treated with corresponding groups. (F) H\&E staining of tumor sections with different groups. All pictures were obtained under the same magnification $(40 \times)$, and the scale bar is $100 \mu \mathrm{m}$. Statistical analysis was conducted via two-tailed paired and unpaired student's $t$ tests to determine differences within groups and between groups, respectively. $P$ value $<0.05$ was considered statistically significant, and the number of the label "** represents the range of $P$ values $(* 0.01<P<0.05 ; * * 0.001<P<0.01$; $* * * P<0.001)$.

biodistribution results, we examined the anticancer efficacy of the NMs in proof-of-concept animal experiments. Mice bearing an MDA-MB-231 tumor of approximately $150 \mathrm{~mm}^{3}$ were divided into five groups $(n=4)$ for various treatments. All of the injections were administrated intravenously (i.v.) every 3 days six times. As exhibited in Figure 6A,B, the mice receiving ICG@ Gel-CuS NMs (NMs/body weight $=50.5 \mathrm{mg} / \mathrm{kg}$ ) did not show any statistically differences in tumor suppression compared with the saline-treated control group. Mice injected with $\mathrm{DOX} \cdot \mathrm{HCl}$ cocktail in saline (DOX/body weight $=2.0 \mathrm{mg} / \mathrm{kg})$ showed a moderate tumor growth suppression $(46.2 \%$ with respect to control), which was restricted by the poor tumor-site accumulation of free chemo-drug (DOX). In particular, considering the nondegradable structure of silica in biophysical circumstance, DOX-loaded silica (DOX@SiO ${ }_{2}$ ) NMs were thus employed for the anticancer treatment investigation. The asprepared DOX@SiO 2 NMs showed spherical morphology with dehydrated size of approximately $100 \mathrm{~nm}$ by TEM and hydrodynamic diameter of $\sim 170 \mathrm{~nm}$ by DLS (Figure S10). Despite the similar morphology and size of DOX@SiO $\mathrm{NMs}_{2}$ to ICG/DOX@Gel-CuS NMs, the multiple injection of DOX@ $\mathrm{SiO}_{2} \mathrm{NMs}(\mathrm{DOX} /$ body weight $=2.0 \mathrm{mg} / \mathrm{kg}$ ) led to moderate tumor inhibition efficacy of $42 \%$, which should be ascribed to the inadequate drug release ability (Figure S11). Remarkably, the tumors of mice received injections of ICG/DOX@Gel-CuS NMs (DOX/body weight $=2.0 \mathrm{mg} / \mathrm{kg}$ ) were efficiently inhibited with $87.8 \%$ of tumor suppression. All of the tumors from various groups were collected and weighed at the end of the treatment (Figure 6C,D), which verified the best therapeutic outcome of the ICG/DOX@Gel-CuS NMs. In addition, H\&E staining of tumor tissue at the end of treatment indicated that most of the tumor cells of the mice treated with ICG/DOX@
Gel-CuS NMs were severely apoptotic and necrotic, while the other groups just exhibited partially damaged tumor tissue (Figure 6F). Taken together, all of the above results documented the distinctive therapeutic efficacy of ICG/ DOX@Gel-CuS NMs benefiting from their enzyme-activated adequate drug release in vivo. Furthermore, to evaluate the safety of the ICG/DOX@Gel-CuS NMs, the mice body weights were recorded throughout the treatment process with weight loss of more than $20 \%$ suggestive of severe adverse side effects. All of the mice from various treatments groups did not display an obvious body weight decrease, and there no statistical significance among the groups (Figure $6 \mathrm{E}$ ). The H\&E staining histologic slices of the major organs showed no significant difference in mice treated with ICG/DOX@Gel-CuS NMs compared with untreated mice (Figure S12). This suggests that the enzyme-responsive ICG/DOX@Gel-CuS NMs are safely administered for tumor therapy.

\section{CONCLUSIONS}

In conclusion, we have successfully constructed ICG/DOX@ Gel-CuS NMs with core-satellite architecture, which conferred fluorescence/PA dual-modal imaging guided enzyme-activated drug release in vivo of the NMs in a real-time and semiquantitative way. The in vitro tests proved that the fluorescence of ICG was initially quenched by the satellite CuS NPs within the intact ICG/DOX@Gel-CuS NMs and showed a nearly linear relationship with the cumulative amount of released DOX from NMs along with the enzyme-triggered degradation. A mathematical model was established via computational simulation to uncover the underlying kinetics functional relationship between the released DOX amount and recovered ICG fluorescence intensity by taking into consideration both the 
processes of NMs erosion and drug diffusion. Additionally, the kinetics of drug release in vivo was assessed by administrating ICG/DOX@Gel-CuS NMs both locally and systemically into tumor-bearing mice. Along with the accumulation of ICG/ DOX@Gel-CuS NMs in the tumor, overexpressed proteases triggered the degradation of the gelatin scaffold as well as the release of DOX and ICG. Based on the established functional relationship between the released DOX amount and ICG fluorescence signal, the kinetic profile of DOX release in vivo of the ICG/DOX@Gel-CuS NMs was successfully derived and monitored quantitatively by fluorescence imaging. Additionally, the photoacoustic signal from $\mathrm{CuS}$ NPs was independent from the physical status of ICG/DOX@Gel-CuS NMs and, hence, was utilized for real-time NMs tracking.

\section{EXPERIMENTAL METHODS}

1. Materials. Gelatin type A from porcine skin (gel strength $\sim 300 \mathrm{~g}$ of Bloom), sulfur powder (99.98\% trace metals basis), and copper(I) chloride (99.999\% metals basis) were purchased from Alfa Aesar (Thermo Fisher Scientific, Heysham, U.K.). Indocyanine Green (ICG) was purchased from TCI Development Co., Ltd. (Shanghai, China). Mercapto-polyethylene glycol-amino (SH-PEG-NH $\left.{ }_{2}, 2 \mathrm{kDa}, 95 \%\right)$ and methyl-polyethylene glycol-amine (mPEG- $\left.\mathrm{NH}_{2}, 5 \mathrm{kDa}, 95 \%\right)$ were purchased from Yare Biotech Co. Ltd. (Shanghai, China). Glutaraldehyde solution (50\%), oleylamine $(80-90 \%)$, and octadecene (90\%) were purchased from Sinopharm Chemical Reagent Co., Ltd. (Shanghai, China). N-Hydroxysuccinimide (NHS, 98\%) and $\mathrm{N}$-(3(dimethylamino)propyl)- $N^{\prime}$-ethylcarbodiimide hydrochloride (EDC, $98 \%$ ) were purchased from Sigma-Aldrich Co. (Saint Louis, MO). Fetal bovine serum (FBS), trypsin-EDTA solution (0.25\%), penicillinstreptomycin solution, and Dulbecco's Modified Eagle's Medium (DMEM) were purchased from Gibco Life Technologies (Thermo Fisher Scientific). All chemicals were of analytical grade and used as received.

2. Preparation of ICG- and DOX-Loaded Gelatin Nanoparticles (ICG/DOX@Gel NPs). Gelatin nanoparticles (Gel NPs) were fabricated by a modified two-step desolvation method. Briefly, gelatin type A $(625 \mathrm{mg})$ was dissolved into $12.5 \mathrm{~mL}$ of deionized water at $40{ }^{\circ} \mathrm{C}$. Next, $12.5 \mathrm{~mL}$ of acetone was added dropwise $(6 \mathrm{~mL} / \mathrm{min})$ into continuously stirring $(600 \mathrm{rpm})$ gelatin solution for $10 \mathrm{~min}$. Next, the supernatant containing the low molecular weight gelatin fraction was removed. Approximately half of the solution was removed, and the $\mathrm{pH}$ of the remaining solution was adjusted to $\sim 2.5$ by addition of $1 \mathrm{M}$ $\mathrm{HCl}$. Next, $20.75 \mathrm{~mL}$ of acetone was added dropwise $(1 \mathrm{~mL} / \mathrm{min})$ into the solution under continuous stirring $(1000 \mathrm{rpm})$. To cross-link the gelatin nanoparticles, $1 \mathrm{~mL}$ of acetone containing $30 \mu \mathrm{L}$ of $50 \%$ glutaraldehyde solution was added dropwise $(0.05 \mathrm{~mL} / \mathrm{min})$ into the solution at $40{ }^{\circ} \mathrm{C}$ under constant stirring $(1000 \mathrm{rpm})$. After $16 \mathrm{~h}$, the acetone and the excess of glutaraldehyde were removed by rotary evaporation and the volume of the solution was decreased to $\sim 5 \mathrm{~mL}$. The solution was purified by dialysis against water to remove remaining acetone and glutaraldehyde. Then the solution was diluted into $50 \mathrm{~mL}$. To fabricate ICG and DOX-load gelatin NPs (ICG/DOX@Gel), 2.5 $\mathrm{mL}$ of Gel NPs solution, $0.125 \mathrm{~mL}$ of ICG solution $(1 \mathrm{mg} / \mathrm{mL}), 1 \mathrm{~mL}$ of DOX solution $(5 \mathrm{mg} / \mathrm{mL})$, and $1.375 \mathrm{~mL}$ of $\mathrm{ddH}_{2} \mathrm{O}$ were mixed under sonication for $30 \mathrm{~min}$. Finally, the solution was centrifuged to remove free ICG and DOX, and the precipitate was dissolved in PBS (1X, pH 7.4).

3. Preparation of ICG/DOX@Gel-CuS NPs. First, $1 \mathrm{~mL}$ of ICG/ DOX@Gel $(10 \mathrm{mg} / \mathrm{mL})$ in PBS, $2 \mathrm{mg}$ of EDC, and $2 \mathrm{mg}$ of NHS were mixed under continuous stirring for $30 \mathrm{~min}$ at $\mathrm{pH} \sim 6$ to activate the carboxyl on the surface of gelatin nanoparticles. Next, the above solution was adjusted to $\mathrm{pH} \sim 7.2$ and dropwise added into the PEGmodified CuS NP aqueous solution $\left(1.004 \times 10^{-6} \mathrm{M}\right)$. The mixture was continuously stirred for $2 \mathrm{~h}$ at constant temperature. Next, $0.2 \mathrm{~mL}$ of EDC stock solution $(20 \mathrm{mg} / \mathrm{mL})$ and $0.2 \mathrm{~mL}$ of NHS stock solution $(20 \mathrm{mg} / \mathrm{mL})$ were added to the ICG/DOX@Gel-CuS solution at $\mathrm{pH}$ of $\sim 6$ and kept for $30 \mathrm{~min}$. The $\mathrm{pH}$ of the mixture was brought to $\sim 7.2$, and $0.05 \mathrm{~mL}$ of water containing $20 \mathrm{mg}$ of methyl-PEG-amine (PEG$\mathrm{NH}_{2}, 5 \mathrm{kDa}$ ) was added into the mixture of ICG/DOX@Gel-CuS. The reaction was processed at room temperature for $2 \mathrm{~h}$. Finally, the obtained ICG/DOX@Gel-CuS NPs were purified by three rounds of ultracentrifugation.

4. Release of ICG and DOX from ICG/DOX@Gel-CuS Dispersed in a Buffer Solution. ICG/DOX@Gel-CuS was dispersed in PBS at an ICG concentration of $5 \mu \mathrm{g} / \mathrm{mL}$ at $37^{\circ} \mathrm{C}, 10 \mu \mathrm{L}$ of $0.25 \%$ trypsin were added into $1 \mathrm{~mL}$ of ICG/DOX@Gel-CuS dispersion, and the hydrodynamic diameter (HD) was monitored by dynamic light scattering (DLS, model Zetasizer Nano ZS, Malvern Panalytical Ltd., Malvern, U.K.) at different time points. The morphology ICG/DOX@ Gel-CuS was assessed by transmission electron microscopy (TEM, model HT7700, Hitachi Ltd., Tokyo, Japan). The fluorescence was recorded by fluorescence spectrophotometry (model F-7000, Hitachi Ltd.) and in vivo fluorescence imaging (model Maestro 2, Cambridge Research \& Instrumentations, Inc., Woburn, MA). To assess the profile of DOX released from ICG/DOX@Gel-CuS, the NMs were centrifuged, and the supernatant was collected for quantification of free DOX with UV at different times.

5. Animal Tumor Model. MDA-MB-231 cells were cultured in DMEM medium supplemented with $10 \%$ FBS, $100 \mu \mathrm{g} / \mathrm{mL}$ streptomycin, and $100 \mathrm{U} / \mathrm{mL}$ penicillin. Cells were grown in tissue culture flasks in a humidified atmosphere containing $5 \% \mathrm{CO}_{2}$ at $37{ }^{\circ} \mathrm{C}$. Female BALB/c nude mice (4-6 weeks old) were supplied from Vital River Laboratory Animal Technology Co., Ltd. (Beijing, China). The tumor animal model was obtained by injecting $\sim 5 \times 10^{6}$ MDA-MB-231 cells in $100 \mu \mathrm{L}$ of PBS into the right flank of the mice. When the tumor volume reached $150 \mathrm{~mm}^{3}$, the imaging and therapy experiments were carried out in accordance with the guidelines approved by the Peking University ethics committee. In antitumor treatment evaluation in vivo, the dose of DOX- or DOX-loaded nanoparticles are equal to $2 \mathrm{mg} / \mathrm{kg}$, the treatment was conducted every 3 days six times. Tumor volume and body weight were recorded every 3 days until the end of 3 weeks. The formula of tumor volume is "length $\times$ width $^{2} / 2$ ".

6. In Vivo Imaging for Drug Release from ICG/DOX@Gel-CuS. When the tumor volume reached $150 \mathrm{~mm}^{3}$, ICG/DOX@Gel-CuS $(2.750 \mathrm{mg} / \mathrm{mL}, 25 \mu \mathrm{L})$ was injected both directly into the tumor and subcutaneously on the left flank in a group of tumor-bearing mice $(n=$ 3 ). The mice were imaged by in vivo fluorescence imaging at different time points postinjection. Another group of tumor-bearing mice were divided into two subgroups $(n=3)$ and intravenously injected with free ICG and ICG/DOX@Gel-CuS NPs, respectively, in saline (200 uL, 50 $\mu \mathrm{g} / \mathrm{mL}$ of ICG). The mice were imaged by in vivo fluorescence imaging and multispectral optoacoustic tomography (MSOT, model in Vision 128, iThera Medical GmbH, Munich, Germany) at different time points postinjection.

\section{ASSOCIATED CONTENT}

\section{S Supporting Information}

The Supporting Information is available free of charge on the ACS Publications website at DOI: 10.1021/acsnano.8b05136.

Materials information, the preparation method of PEGmodified CuS NPs and DOX@SiO 2 NPs, movie information, and statistical analysis (PDF)

Spatial-temporal dynamic process of the DOX release from NMs in response to trypsin (AVI)

Spatial-temporal dynamic change of the fluorescence intensity during the degradation of NMs in response to trypsin (AVI)

3D-reconstruction of transillumination fluorescence images of mice treated with NMs via tail vein injection at $2 \mathrm{~h}$ post-injection (AVI)

3D-reconstruction of transillumination fluorescence images of mice treated with NMs via tail vein injection at $4 \mathrm{~h}$ post-injection (AVI) 
3D-reconstruction of transillumination fluorescence images of mice treated with NMs via tail vein injection at $24 \mathrm{~h}$ post-injection (AVI)

\section{AUTHOR INFORMATION}

\section{Corresponding Authors}

*E-mail: wuy@nanoctr.cn.

*E-mail: tjuguoweisheng@126.com.

*E-mail: liangxj@nanoctr.cn.

\section{ORCID $\odot$}

Yan Wu: 0000-0001-8508-7077

Weisheng Guo: 0000-0002-8064-357X

Xing-Jie Liang: 0000-0002-4793-1705

\section{Author Contributions}

${ }^{\otimes}$ X.L. and M.B. contributed equally to this work.

\section{Notes}

The authors declare no competing financial interest.

\section{ACKNOWLEDGMENTS}

This work was supported by the National Natural Science Foundation Key Projects (Nos. 31630027, 31430031, 81601603, 81773185, and 81471739), NSFC-DFG project (No. 31761133013), and the National Distinguished Young Scholars grant (No. 31225009). The authors also appreciate the support by the external cooperation program of the Chinese Academy of Science (No. 121D11KYSB20160066), the "Strategic Priority Research Program" of the Chinese Academy of Sciences (Grant No. XDA09030301), the National Key Research Program of China (Grant Nos. 2016YFA0100900 and 2016YFA0100902), and the NanOArt grant of the "Mission Sustainability" of the University of Rome Tor Vergata.

\section{REFERENCES}

(1) Shi, J.; Kantoff, P. W.; Wooster, R.; Farokhzad, O. C. Cancer Nanomedicine: Progress, Challenges and Opportunities. Nat. Rev. Cancer 2017, 17, 20-37.

(2) Leggett, S. E.; Wong, I. Y. Nanomedicine: Catching Tumour Cells in the Zone. Nat. Nanotechnol. 2017, 12, 191-193.

(3) Chen, Q.; Du, Y.; Zhang, K.; Liang, Z.; Li, J.; Yu, H.; Sun, J. TauTargeted Multifunctional Nanocomposite for Combinational Therapy of Alzheimer's Disease. ACS Nano 2018, 12, 1321-1338.

(4) He, S.; Li, C.; Zhang, Q.; Ding, J.; Liang, X. J.; Chen, X.; Huang, Y. Tailoring Platinum (IV) Amphiphiles for Self-Targeting All-in-One Assemblies as Precise Multimodal Theranostic Nanomedicine. ACS Nano 2018, 12, 7272-7281.

(5) Zhang, H.; Zhu, Y.; Qu, L.; Wu, H.; Kong, H.; Yang, Z.; Hai, M. Gold Nanorods Conjugated Porous Silicon Nanoparticles Encapsulated in Calcium Alginate Nano Hydrogels Using Microemulsion Templates. Nano Lett. 2018, 18, 1448-1453.

(6) Zhou, T.; Du, Y.; Wei, T. Transcriptomic Analysis of Human Breast Cancer Cells Reveals Differentially Expressed Genes and Related Cellular Functions and Pathways in Response to Gold Nanorods. Biophys. Rep. 2015, 1, 106-114.

(7) Jiang, W.; von Roemeling, C. A.; Chen, Y.; Qie, Y.; Liu, X.; Chen, J.; Kim, B. Y. Designing Nanomedicine for Immuno-Oncology. Nat. Biomed Eng. 2017, 1, 0029.

(8) Jiang, W.; Yuan, H.; Chan, C. K.; von Roemeling, C. A.; Yan, Z.; Weissman, I. L.; Kim, B. Y. Lessons from Immuno-Oncology: A New Era for Cancer Nanomedicine. Nat. Rev. Drug Discovery 2017, 16, 369.

(9) Xue, X.; Zhao, Y.; Zhang, X.; Zhang, C.; Kumar, A.; Zhang, X.; Liang, X. J. Phenylboronic Acid-Functionalized Magnetic Nanoparticles for One-Step Saccharides Enrichment and Mass Spectrometry Analysis. Biophys. Rep. 2015, 1, 61-70.
(10) Nel, A.; Ruoslahti, E.; Meng, H. New Insights into "Permeability" as in the Enhanced Permeability and Retention Effect of Cancer Nanotherapeutics. ACS Nano 2017, 11, 9567-9569.

(11) Wang, L.; Huang, J.; Chen, H.; Wu, H.; Xu, Y.; Li, Y.; Mao, H. Exerting Enhanced Permeability and Retention Effect Driven Delivery by Ultrafine Iron Oxide Nanoparticles with $\mathrm{T} 1-\mathrm{T} 2$ Switchable Magnetic Resonance Imaging Contrast. ACS Nano 2017, 11, 45824592.

(12) Iyer, A. K.; Khaled, G.; Fang, J.; Maeda, H. Exploiting the Enhanced Permeability and Retention Effect for Tumor Targeting. Drug Discovery Today 2006, 11, 812-818.

(13) Koudelka, S.; Turánek, J. Liposomal Paclitaxel Formulations. J. Controlled Release 2012, 163, 322-334.

(14) Mandal, A.; Bisht, R.; Rupenthal, I. D.; Mitra, A. K. Polymeric Micelles for Ocular Drug Delivery: from Structural Frameworks to Recent Preclinical Studies. J. Controlled Release 2017, 248, 96-116.

(15) Su, Y.; Wang, K.; Li, Y.; Song, W.; Xin, Y.; Zhao, W.; Lu, L. Sorafenib-Loaded Polymeric Micelles as Passive Targeting Therapeutic Agents for Hepatocellular Carcinoma Therapy. Nanomedicine 2018, 13, $1009-1023$

(16) Park, J.; Park, J. E.; Hedrick, V. E.; Wood, K. V.; Bonham, C.; Lee, W.; Yeo, Y. A Comparative In Vivo Study of Albumin-Coated Paclitaxel Nanocrystals and Abraxane. Small 2018, 14, 1703670.

(17) Tan, Y. L.; Ho, H. K. Navigating Albumin-Based Nanoparticles through Various Drug Delivery Doutes. Drug Discovery Today 2018, 23, $1108-1114$

(18) Anchordoquy, T. J.; Barenholz, Y.; Boraschi, D.; Chorny, M.; Decuzzi, P.; Dobrovolskaia, M. A.; Godin, B. Mechanisms and Barriers in Cancer Nanomedicine: Addressing Challenges. ACS Nano 2017, 11, $12-18$.

(19) Rosenblum, D.; Joshi, N.; Tao, W.; Karp, J. M.; Peer, D. Progress and Challenges towards Targeted Delivery of Cancer Therapeutics. Nat. Commun. 2018, 9, 1410.

(20) White, S. C.; Lorigan, P.; Margison, G. P.; Margison, J. M.; Martin, F.; Thatcher, N.; Ranson, M. Phase II Study of SPI-77 (Sterically Stabilised Liposomal Cisplatin) in Advanced Non-SmallCell Lung Cancer. Br. J. Cancer 2006, 95, 822.

(21) Friedman, A. B.; Brown, S. J.; Bampton, P.; Barclay, M. L.; Chung, A.; Macrae, F. A.; Sparrow, M. P. Randomised Clinical Trial: Efficacy, Safety and Dosage of Adjunctive Allopurinol in Azathioprine/ Mercaptopurine Nonresponders (AAA Study). Aliment. Pharmacol. Ther. 2018, 47, 1092-1102.

(22) Darwich, A. S.; Ogungbenro, K.; Vinks, A. A.; Powell, J. R.; Reny, J. L.; Marsousi, N.; McCune, J. S. Why Has Model-Informed Precision Dosing not yet Become Common Clinical Reality? Lessons from the Past and A Roadmap for the Future. Clin. Pharmacol. Ther. 2017, 101, 646-656.

(23) Wang, R.; Zhou, L.; Wang, W.; Li, X.; Zhang, F. In Vivo Gastrointestinal Drug-Release Monitoring through Second NearInfrared Window Fluorescent Bioimaging with Orally Delivered Microcarriers. Nat. Commun. 2017, 8, 14702.

(24) Fan, Z.; Sun, L.; Huang, Y.; Wang, Y.; Zhang, M. Bioinspired Fluorescent Dipeptide Nanoparticles for Targeted Cancer Cell Imaging and Real-Time Monitoring of Drug Release. Nat. Nanotechnol. 2016, $11,388$.

(25) Liu, J.; Bu, J.; Bu, W.; Zhang, S.; Pan, L.; Fan, W.; Du, J. RealTime In Vivo Quantitative Monitoring of Drug Release by Dual-Mode Magnetic Resonance and Upconverted Luminescence Imaging. Angew. Chem., Int. Ed. 2014, 53, 4551-4555.

(26) Wong, P. T.; Choi, S. K. Mechanisms of Drug Release in Nanotherapeutic Delivery Systems. Chem. Rev. 2015, 115, 3388-3432. (27) Xue, X.; Jin, S.; Zhang, C.; Yang, K.; Huo, S.; Chen, F.; Liang, X. J. Probe-Inspired Nano-Prodrug with Dual-Color Fluorogenic Property Reveals Spatiotemporal Drug Release in Living Cells. ACS Nano 2015, 9, 2729-2739.

(28) Luo, D.; Goel, S.; Liu, H. J.; Carter, K. A.; Jiang, D.; Geng, J.; Fang, C. Intrabilayer ${ }^{64} \mathrm{Cu}$ Labeling of Photoactivatable, DoxorubicinLoaded Stealth Liposomes. ACS Nano 2017, 11, 12482-12491. 
(29) Jiang, Q.; Nie, Y.; Chen, X.; He, Y.; Yue, D.; Gu, Z.pH-Triggered Pinpointed Cascading Charge-Conversion and Redox-Controlled Gene Release Design: Modularized Fabrication for Nonviral Gene Transfection. Adv. Funct. Mater. 2017, 27, 1701571.

(30) Li, S. Y.; Liu, L. H.; Rong, L.; Qiu, W. X.; Jia, H. Z.; Li, B.; Zhang, X. Z. A Dual-FRET-Based Versatile Prodrug for Real-Time Drug Release Monitoring and In Situ Therapeutic Efficacy Evaluation. Adv. Funct. Mater. 2015, 25, 7317-7326.

(31) Guo, X.; Wang, L.; Duval, K.; Fan, J.; Zhou, S.; Chen, Z. Dimeric Drug Polymeric Micelles with Acid-Active Tumor Targeting and FRET-Traceable Drug Release. Adv. Mater. 2018, 30, 1705436.

(32) Yang, G.; Xu, L.; Xu, J.; Zhang, R.; Song, G.; Chao, Y.; Liu, Z. Smart Nanoreactors for $\mathrm{pH}$-Responsive Tumor Homing, Mitochondria-Targeting, and Enhanced Photodynamic-Immunotherapy of Cancer. Nano Lett. 2018, 18, 2475-2484.

(33) Jia, X.; Zhang, Y.; Zou, Y.; Wang, Y.; Niu, D.; He, Q.; Li, Y. Dual Intratumoral Redox/Enzyme-Responsive NO-Releasing Nanomedicine for the Specific, High-Efficacy, and Low-Toxic Cancer Therapy. Adv. Mater. 2018, 30, 1704490.

(34) Mu, J.; Lin, J.; Huang, P.; Chen, X. Development of Endogenous Enzyme-Responsive Nanomaterials for Theranostics. Chem. Soc. Rev. 2018, 47, 5554-5573.

(35) Coester, C. J.; Langer, K.; Von Briesen, H.; Kreuter, J. Gelatin Nanoparticles by Two Step Desolvation a New Preparation Method, Surface Modifications and Cell Uptake. J. Microencapsulation 2000, 17, 187-193.

(36) Cohen, M. H.; Turnbull, D. Molecular Transport in Liquids and Glasses. J. Chem. Phys. 1959, 31, 1164-1169.

(37) Amsden, B. Solute Diffusion within Hydrogels. Mechanisms and Models. Macromolecules 1998, 31, 8382-8395.

(38) Lustig, S. R.; Peppas, N. A. Solute Diffusion in Swollen Membranes. IX. Scaling Laws for Solute Diffusion in Gels. J. Appl. Polym. Sci. 1988, 36, 735-747.

(39) Brown, E.; McKee, T.; Pluen, A.; Seed, B.; Boucher, Y.; Jain, R. K. Dynamic Imaging of Collagen and Its Modulation in Tumors In Vivo Using Second-Harmonic Generation. Nat. Med. 2003, 9, 796.

(40) Kessenbrock, K.; Plaks, V.; Werb, Z. Matrix Metalloproteinases: Regulators of the Tumor Microenvironment. Cell 2010, 141, 52-67.

(41) Lai, Y. H.; Chiang, C. S.; Kao, T. H.; Chen, S. Y. Dual-Drug Nanomedicine with Hydrophilic F127-Modified Magnetic Nanocarriers Assembled in Amphiphilic Gelatin for Enhanced Penetration and Drug Delivery in Deep Tumor Tissue. Int. J. Nanomed. 2018, 13, 3011.

(42) Wong, C.; Stylianopoulos, T.; Cui, J.; Martin, J.; Chauhan, V. P.; Jiang, W.; Fukumura, D. Multistage Nanoparticle Delivery System for Deep Penetration into Tumor Tissue. Proc. Natl. Acad. Sci. U. S. A. 2011, 108, 201018382. 